\title{
Patient feedback on their cancer care experiences analyzed using the Cancer Experience Measurement Framework
}

Michaela Ann Bourque ( $\sim$ michaela.bourque@mail.mcgill.ca )

McGill University https://orcid.org/0000-0002-1926-0975

Carmen Loiselle

McGill University

\section{Research Article}

Keywords: Patient experiences, Patient satisfaction, Cancer care, Content analysis, Person-centered care, patient experience measurement framework

Posted Date: February 25th, 2022

DOI: https://doi.org/10.21203/rs.3.rs-982308/v1

License: (c) (i) This work is licensed under a Creative Commons Attribution 4.0 International License.

Read Full License 


\section{Abstract}

Background: Recent advances in research on patient experiences with cancer underscore the importance of proactively seeking feedback from users of cancer care services.

Methods: The present study administered the Ambulatory Oncology Patient Satisfaction Survey (AOPSS) with 7,885 questionnaire packets mailed out to potential participants. 3,279 completed surveys were returned and 692 of these contained feedback to an open-ended question about patients' perceptions of the cancer care they had received within the last 6 months since diagnosis.

Results: Using the Cancer Experience Measurement Framework (CEMF) to guide the in-depth thematic analysis of patient feedback, 19 key themes emerged, some of which include: treatment setting, time, location, service availability, perceived appropriateness, personnel or specialists involved, patient preferences, information/education, continuity/transition of care, coordination of care, access to care, wait times, access to emergency rooms, and intensive care unit visits.

Conclusions: Findings suggest that patient's dissatisfaction is heightened in settings high in perceived disorganization. Participants suggest that improving wait times in both ambulatory and emergency settings, in addition to ensuring smooth access and coordination of care both intra- and interorganization cancer care services across the illness trajectory is necessary for creating a more personcentred care experience. These findings are timely as they underscore how informative patient voices are to ensure that cancer settings provide quality, timely, and personalized care that patients have come to expect.

\section{Background}

A cancer diagnosis and its treatment come with a host of challenges filtered through the lenses of personal experience with cancer, exchanges with significant others, and interactions with the health care system (Loiselle et al., 2019). As such, the cancer experience is highly dynamic, involving various interactions, events and transitions across the cancer trajectory. These experiences can be optimized through reliance on situation-responsive and personalized person-centred care (PCC) approaches (Loiselle \& Brown, 2020). Defined as respectful, active, and tailored approaches that meet patients' needs, values, and ideals, PCC serves as a primary determinant of overall quality of care, including more positive interactions with healthcare professionals and optimal health-related outcomes (Loiselle et al., 2019; Grover et al., 2018).

Wong and colleagues (2015) note that direct feedback from users (e.g., patients) is best for assessing patients' interactions with healthcare. Patient-focused questionnaires, for instance, capture elements (good and bad) of healthcare systems that are important to patients. Self-report data are key when assessing cancer care services and patients' outcomes while also being highly relevant to refine care practices. Reliance on standardized patient experience questionnaires allows a deeper and more thorough understanding of users' experiences within and across respondents (Wong et al., 2015). Bridge 
and colleagues (2019), for instance, have used a standardized questionnaire (the Ambulatory Oncology Patient Satisfaction Survey, AOPSS) document outpatient cancer care experiences. Schoen et al. (2005) note that patient self-report data are also crucial in making international comparisons across care services and identifying areas for improvement. However, key themes and patient experience categories are often suggested by surveys (top down) rather than emerging from feedback provided by patients themselves (bottom up). Hence, the need for the present study.

\section{Mapping out people's experiences with cancer: The Cancer Experience Measurement Framework}

Evidence suggests that patients encounter multidimensional challenges related to their healthcare experiences (Pelzang, 2010; Bridge et al., 2019). Lee et al. (2010), for instance, note that the primary goal of care coordination is to optimize outcomes through timely health service delivery (e.g., better access, more relevant interventions, and higher patient satisfaction). However, important aspects of cancer care, as reported by users, have not been studied extensively.

The Cancer Experience Measurement Framework (CEMF; Loiselle et al., 2019) stems from an extensive review of the current literature on the experiential context of individuals affected by cancer. The CEMF proposes four main categories that must be attended if seeking cancer experiences in their globality: the patient perspective, the family perspective, the shared patient-family perspective, and users' interactions with the healthcare system (Loiselle et al., 2019). Interactions with healthcare (the focus of this paper) encompass several facets, including the structure, processes, and outcomes related to cancer care services. Access to and patient satisfaction with these services is most informative (Loiselle \& Brown, 2020; Loiselle et al., 2019). The following applies the CEMF to the voluntary reported experiences of those having received cancer care.

\section{Methods}

\section{Sample}

The sample consisted of patients with diverse cancer diagnoses, aged 18 years or older, and treated for cancer within the past six months. A total of 7,885 surveys were mailed out over four years ending in 2019. In total, 3,279 completed surveys were returned for a response rate of $41.6 \%$ (Loiselle et al., 2020). Of these, 692 responded to the open-ended portion of AOPSS.

\section{Setting}

The survey took place in three university-affiliated hospitals with integrated cancer centers in Montreal, Quebec, Canada. These included the Segal Cancer Centre at the Jewish General Hospital (CIUSSS CentreOuest), the Cedars Cancer Centre at the McGill University Health Centre, and the Cancer Centre at St. Mary's Hospital (CIUSSS Ouest) (see also Loiselle et al., 2020).

\section{Design and measures}


The study employed a mailed-out large-scale survey design. The Ambulatory Oncology Patient Satisfaction Survey (AOPSS) is a standardized self-report measure used in healthcare facilities across North America and elsewhere (NRC Picker, National Research Corporation, 2003; Ferguson, 2012). It includes 83 items concerning patient experience and satisfaction; 45 core items address six care domains identified as most important by patients: emotional support, coordination and continuity of care, respect for patient preferences, physical comfort, information/education, and access to care. The survey ends with an open-ended question prompting patients' feedback formulated as "Is there anything else you would like to tell us about your cancer care services?" The Rossy Cancer Network facilitated survey administration, distribution, and compilation with AOPSS license holder NRC Picker.

\section{Procedures}

To meet inclusion criteria, patient health records were cross-referenced with hospital mailing lists to create a random selection of potential participants (RCN, https://www.mcgill.ca/search/Aopss? search_origin=rcr-rcn). After three months, new eligible participants were mailed a patient satisfaction survey package including a cover letter, AOPSS, and a prepaid postage return envelope. Participants were informed that completion and return of the survey indicated voluntary consent. A follow-up letter and additional survey were sent if no completed survey was received approximately four weeks after mailing. No personally identifiable information was collected.

\section{Analysis}

Only data collected from the open-ended AOPSS question "Is there anything else you would like to tell us about your cancer care services?" were used in this analysis. Nearly 700 items were analyzed thematically - an approach for identifying and reporting patterns within a given data set (Scharp \& Sanders, 2019). Each response was first classified as positive, negative, mixed, or neutral. Responses classified as neutral (e.g., "No, thank you" or "Not at this time") were excluded from further analysis. Data were subsequently coded according to the six AOPSS care domains of emotional support, coordination and continuity of care, respect for patient preferences, physical comfort, information and education, and access to care (Loiselle et al., 2020; Gerteis et al., 1993; Davis et al., 2005). Analysis showed feedback related to the nature of care received, access and continuity of cancer care, and care satisfaction - all elements proposed by the CEMF focusing on patient interactions with healthcare systems (Loiselle et al., 2019).

\section{Results}

Of 692 responses received, 45 were excluded due to their neutral nature, leaving 647 responses for analysis. $93.5 \%$ of participant feedback contained one or more components tied to at least one facet of the CEMF. Skewed percentages of positive $(n=352,54 \%)$ to negative feedback $(n=145,23 \%)$ and mixed $(n=145,23 \%)$ responses provided nuanced accounts concerning the nature of cancer care services received. 


\section{Descriptive Statistics}

Nearly $60 \%$ of respondents were female, and $51 \%$ of all participants reported being over 65 (Rossy Cancer Network, 2019). Various cancers were represented, the most common being breast cancer (29\%), followed by hematology/lymphoma (14\%). 156 participants did not report a specific diagnosis. Of the 3,278 completed surveys, 692 answered the final open-ended question, representing a $21 \%$ response rate for this item. Respondents are distributed as follows: Segal Cancer Centre (34\%), Cedars Cancer Centre (37\%) and the Cancer Centre at St. Mary's General Hospital (29\%).

\section{Interactions with Healthcare Systems - Access and Coordination of Care}

Of 692 respondents, $284(41 \%)$ mentioned access or coordination of care issues, with $105(37 \%)$ of these considered positive, 65 (23\%) negative, and 114 (40\%) mixed. Further analysis classified responses according to CEMF core domains of patient interactions with healthcare systems: structure, processes of care, utilization, and patient satisfaction (Loiselle et al., 2019). Examples of verbatim feedback follow.

\section{Domain 1: Structure of the health care system}

This domain represents material, human, and organizational resources that affect health care systems' ability to provide individualized/personalized care (Loiselle et al., 2019). Subcategories of this include setting, time, location, service availability, perceived appropriateness, and personnel or specialists involved (Loiselle et al., 2019).

\section{Negative feedback.}

"I had weekly chemo treatments (IV) \& it was necessary to have a blood test the day before the treatment. I understand it had to be done, but this meant 2 visits weekly to [the cancer centre] \& sometimes it was inconvenient, time-consuming - also paying for parking twice weekly!' (Comment 59; Relevant Themes: time, setting, perceived appropriateness, availability of care)

\section{Positive feedback.}

"I am very pleased with the cancer care I am receiving. The doctor and clinical team are doing an excellent job. I am already surviving for 10 years. The wait times are always acceptable, very rarely too long. The team and oncologist are very professional. I fully understand the treatment I am receiving. The pharmacist is also always very accessible and helpful. I am very grateful for my treatments. Thanks so much." (Comment 286; Relevant Themes: time, perceived appropriateness, personnel involved, availability of care)

\section{Mixed feedback.}

"[An] excellent cancer care team at [the cancer centre] (receptionists, secretaries, technologists, doctors, etc). Very compassionate and professional. Extremely competent. An area that can be improved [is] the ability to reach receptionists/secretaries to schedule appointments or get updates. Typically, I was 
diverted to voicemails, and sometimes my calls were not returned." (Comment 662; Relevant Themes: time, perceived appropriateness, personnel involved, availability of care)

\section{Domain 2: Processes of Care}

Care processes represent the interwoven series of events that contribute to individualized patient care experiences, for example, timelines related to diagnosis, planning and implementation of treatment regimens (Loiselle et al., 2019). Care process outcomes represent effects related to system services such as cancer morbidity, adverse events, cancer-related hospital (re) admissions, and emergency room visits (Loiselle et al., 2019). Domain subcategories include patient preferences, emotional support, physical comfort, information/education, continuity/transition, coordination of care, access to care, and family \& friends (Loiselle et al., 2019).

\section{Negative feedback.}

"Very trying experience... Had to wait very long for diagnosis... Oncologist very professional but gave rehearsed answers, did not understand or care about my feelings of fear and apprehension. [I] felt very much like a number. For emergencies unable to contact anyone after $4 \mathrm{pm}$ or on the weekends. [As a result, I] had to wait over 17 hours in [the] emergency room!!! - Absolutely no info about alternative therapies!" (Comment 72; Relevant Themes: patient preferences, information/education, continuity/transition, coordination of care, access to care, emotional support)

\section{Positive feedback.}

"I think the cancer wards/rooms are exceptional. I think the staff is kind \& patient. In particular, the staff at the main reception counters. There are also volunteers to answer any questions we may have. My oncologist \& pivot nurse are also exceptional - I am positive I am in the right place for treatment. No one wants or deserves this illness but having health care that [is] such great support certainly facilitates the journey..." (Comment 381; Relevant Themes: patient preference, physical comfort, emotional support, information/education, access to care, coordination of care)

\section{Mixed feedback.}

"The service was very good. The staff at radiation oncology were very gentle, compassionate, punctual, respectful + polite. They explained very well if I had questions. Unfortunately, the secretaries forget to do appointments and then when you are there, they shuffle the charts, [so] you think there is one or two before you and suddenly there are 4 or 5 [other patients] before you." (Comment 607; Relevant Themes: emotional support, physical comfort, patient preference, information/education, access to care, coordination of care)

\section{Domain 3: Health care Utilization}


This domain represents the accessibility of healthcare services and facilities. These include access to public versus private services, use of hospital equipment (for treatment or further tests), wait times, access to emergency rooms, intensive care unit visits, and referral to relevant programs (Loiselle et al., 2019). This rather specific domain led to few comments; these tended to be negative or mixed.

\section{Negative feedback.}

"Translated from French - [Called the] emergency number in oncology - does not return our calls. I had to go to the hospital emergency room." (Comment 112; Relevant Themes: access to emergency services)

\section{Mixed feedback.}

"... [I] Had to wait very long for a diagnosis. Went to a private breast clinic and spent $\$ 3000$ for diagnosis and biopsy and still had to wait five weeks to see [a] surgeon after a positive diagnosis with Stage III cancer..."(Comment 72; Relevant Themes: wait times, private/public services, referral)

\section{Domain 4: Patient Satisfaction with cancer care}

This domain encompasses patients' and families' expectations, preferences, and perceptions of quality of care (Loiselle et al., 2019). It includes appropriateness, coordination, access, and continuity/transition of care, followed by emotional support and physical comfort (Loiselle et al., 2019). Satisfaction is subjective, stemming from users' desires and expectations (Loiselle et al., 2019). Resultantly, the following comments address users' satisfaction with the setting, wait times, information/education, and other previously mentioned facets of care.

\section{Lower cancer care satisfaction.}

"When a patient is diagnosed with cancer for the first time, the doctors don't explain enough of the situation... they should explain more about cancer because for us it is something new and for us, it is not a daily thing that we know... I hope the system could change so [the patient] could get more information when you are diagnosed with cancer for the first time." (Comment 79; Relevant Themes: information/education, patient preference, expectation)

\section{Higher cancer care satisfaction.}

"Translated from French - I am very satisfied and grateful for the services received. I would recommend your institution to everyone. I appreciated the discussions about taking part in research and possibilities of experimental treatments offered in another university-affiliated cancer centre. I had a great relationship with my 3 treating doctors for 5 years. I thank you greatly for the quality of services offered. During my 2 hospitalizations, the food was excellent." (Comment 410; Relevant Themes: patient preference, expectation, perception of care, information/education)

\section{Mixed cancer care satisfaction.}


"Overall, I am pleased with the care I received and continue to receive. I think there are definite gaps that need to be addressed - in particular, concerning information sharing with the newly diagnosed patient + their families. Also, the doctors need to be more open about complementary + alternative medicine... Nutritionists also need to be trained to consider what is particularly useful to fight cancer. The food I received while in hospital should be banned from cancer wards/patients..." (Comment 582; Relevant Themes: patient preference, expectations, information/education)

\section{Discussion}

The purpose of this study was to analyze voluntary feedback received through a patient satisfaction survey. The CEMF was used to codify these data (Loiselle et al., 2019) in terms of structure, processes of care, utilization, and satisfaction domains of cancer care.

Consistent with the literature (Balogh et al., 2011; Bridge et al., 2019; Loiselle \& Brown, 2020; Loiselle et al., 2019; Loiselle et al., 2020), this analysis supports the diversity of patient perceptions of cancer care. For example, Li and colleagues (2017) note that participants report discrepancies between the nature of the cancer information expected and the information provided when communicating cancer-related information and education. However, to obtain optimal outcomes, cancer information must be tailored appropriately to meet patients' personal, cultural, and health needs (Lyson et al., 2020). In addition, some participants reported being dissatisfied with the amount of cancer-related information provided, whereas others reported receiving more than enough information. This discrepancy may be due, in part, to information/communication preferences, as outlined in Loiselle's (2019) work on patients' distinct preferences for cancer information. According to Hack and colleagues (2006), this discrepancy may also be influenced by patients' preferences for involvement in cancer treatment decision-making.

Longitudinally, women with breast cancer who had greater involvement in decisions regarding their care reported significantly higher overall quality of life (Hack et al., 2006).

Comments directed towards structure, processes of care, and utilization of cancer care services focused heavily on wait times and continuity of care. Like findings by Bridge and colleagues (2019), participants reported dissatisfaction with wait times related to three main areas: waiting to see a healthcare provider, waiting for tests/test results, and waiting for treatment/pharmacy follow-ups. The fourth area underscored time-sensitive cancer-related unmet needs (e.g., no phone calls returned after hour/on weekends from the cancer centre and long wait times in emergency rooms). Furthermore, wait times led some to reach out to private services as more expedient cancer care modalities.

Participants who reported dissatisfaction with continuity of care transitions also felt less emotionally supported throughout the cancer care experience. Like Bridge and Colleagues (2019) and Grunfeld \& Earle (2010), participants also addressed continuity of care and communication among healthcare providers. Specifically, they felt that responsibility should not rest solely on patients to update health care providers about their condition. Instead, they suggested that providers be better equipped to ensure interconnectedness and streamlined information on how treatment and care modalities evolve. Lee 
(2010) notes that coordination issues are especially prevalent for patients whose cancer care is shared by multiple subspecialties and involves multiple services and locations.

Some participants reported that cancer care wait times could lead to avoidable adverse outcomes. One participant noted that "When we have an appointment with the oncologist, the wait can be two hours in the waiting room. Other times we have waited one and a half hours before seeing the oncologist in the cubicle. For a sick patient, this is very difficult considering the side effects due to the treatments". This shared concern should be further addressed clinically and through research.

Participants reported high positive regard for their overall cancer care experience. Indeed, Tremblay and colleagues (2017) found that patients who received care from a high-intensity interdisciplinary team were more likely to report positive experiences in areas like quality of patient-professional communication, continuity of care, and person-centred response. Of interest, positive feedback from this study was most common when referencing interdisciplinary work, with some commenting on being "thankful for the healthcare providers and all they had done," both in terms of physical and emotional support throughout their cancer trajectory. In addition, participants were likely to directly refer to doctors, nurses, and care coordinators as positive figures in their cancer care experience.

Patient preferences seemed to be the most variable facet related to satisfaction with cancer care received. As such, Loiselle and Brown (2020), Gallespie et al. (2018), and Tzelepis et al. (2015) explain that there is no average patient, only individuals whose preferences for care are just that - individual. Participants noted individual preferences such as dates, times, and frequencies of appointments impacting their overall satisfaction.

Supported by the CEMF, this thematic analysis targeted significant areas of the cancer care experience. It provided insight into the multi-faceted nature of cancer care and its positives and negatives as per the patients' perspective. Consistent with previous research, this study has brought back to light the necessity of considering multiple facets of the patient experience when addressing person-centred cancer care.

\section{Limitations}

There are limitations when interpreting the present findings. First, self-selection biases may have been present as participants provided written feedback only if they wish to do so. Depending on their levels of (dis)satisfaction, some individuals may have been more compelled than others to respond to the survey. Due to the retrospective nature of patient experiences (Bridge et al., 2019), recall bias may have been operating. Third, reliance on the CEMF to organize patient feedback may have limited the scope or depth of analysis; an alternate framework may have yielded different interpretations. Last, the fact that some sociodemographic data were not recorded (e.g., sex and language spoken at home) limits the generalizability of findings.

\section{Implications for Future Research}


These findings highlight the need to seriously consider patients' feedback when developing, implementing and revising cancer care practices. As exemplified above, a true person-centred and personalized care approach must periodically query patients about their experiences, preferences and unmet needs. Our own work is a step in that direction (Loiselle \& Brown, 2020; Loiselle, 2019; Loiselle et al., 2019) and future research should continue to gather patient-reported cancer care processes and outcomes, including unaddressed symptoms, urgent care issues, unexplained wait times, and care (dis)continuity. In addition, the Covid-19 pandemic has put undue stress on patient experiences as institutions have shifted dramatically to emphasize infection control over cancer control (Coalition Priorité Cancer Québec, 2020). Processes, such as physical distancing, closing waiting rooms, decreased capacity in medical offices and treatment areas, all have worked to significantly affect people's experiences and outcomes related to cancer.

\section{Conclusion}

Cancer care experiences are multifaceted and dynamic. Interactions with healthcare while coping with cancer have significant ramifications not only for patients' and significant others' experiences but also their health-related outcomes (Loiselle et al., 2019). An in-depth understanding of what cancer care users, rather than institutions, may perceive as most relevant and timely is vital (Bridge et al., 2019; Loiselle et al., 2019; Loiselle \& Brown, 2020). By conducting a thematic analysis based on structure, processes, utilization, and satisfaction domains, various patient experience factors emerged. In accordance, improving wait times in ambulatory and emergency settings, ensuring smooth coordination of care and access to intra- and inter-organization cancer care services across the illness trajectory are priorities. In addition, a deeper examination of potential adverse cancer outcomes linked to systems-related factors is long overdue.

\section{Abbreviations}

AOPSS - Ambulatory Oncology Patient Satisfaction Survey

CEMF - Cancer Experience Measurement Framework

\section{Declarations}

Ethics Approval and Consent to participate: This study was done as a quality assurance study and therefore did not require ethics approval. Informed consent was obtained through the statement provided to all participants: "Questionnaire completion and return signify consent to take part in the study".

Consent for publication: Not Applicable

Availability of data and material: Data are available upon request to Michaela Bourque by email Michaela.bourque@mail.mcgill.ca 


\section{Competing interests: None}

Funding: This was a third-party funder. There is no conflict of interest in the funding and instrumental assistance they provided to conduct the survey.

Authors Contributions: The senior author (CGL) contributed to study conception, design, and implementation. Data management and analysis were performed by the first author (MAB) with supervision by the senior author. All authors were involved in the write-up of the manuscript. All authors approve the final manuscript. We thank Dr. Tyler Brown for comments on earlier versions of the manuscript.

Acknowledgements: The present study would not have been possible without the engagement of participants who generously took the time to complete study materials. Gratitude is owed to the Rossy Cancer Network (for the financial support of survey administration) and the three participating cancer centres for providing logistic support. Last, thanks go to Rachel Leland, Dr. Elissa Rodkey, Dr. Tyler Brown and additional reviewers for their feedback, advice, and support at various stages of manuscript preparation.

\section{References}

1. Balogh, E. P., Ganz, P. A., Murphy, S. B., Nass, S. J., Ferrell, B. R., \& Stovall, E. (2011). Patient-centered cancer treatment planning: improving the quality of oncology care. Summary of an Institute of Medicine workshop. The oncologist, 16(12), 1800-1805.

https://doi.org/10.1634/theoncologist.2011-0252

2. Bridge, E., Conn, L. G., Dhanju, S., Singh, S., \& Moody, L. (2019). The patient experience of ambulatory cancer treatment: a descriptive study. Current Oncology (Toronto, Ont.), 26(4), e482-e493. https://doi.org/10.3747/co.26.4191

3. Coalition Priorité Cancer Quebec. (2020). The Impact of the Measures Implemented to Counter the COVID-19 Pandemic on Oncology Patients. https://coalitioncancer.com/wpcontent/uploads/2020/06/FINAL_REPORT_COVID-CANCER-JUNE2020.pdf

4. Davis, K., Schoenbaum, S. C., \& Audet, A. M. (2005). A 2020 vision of patient-centered primary care. Journal of general internal medicine, 20(10), 953-957. https://doi.org/10.1111/j.15251497.2005.0178.x

5. Ferguson, D. (2012). Validation of the NRC Picker Canada Ambulatory Oncology Patient Satisfaction Survey. Ontario: NRC Picker Canada.

6. Gerteis M, Edgman-Levitan S, Daley J, Delbanco TL. (1993). Through the patient's eyes: Understanding and promoting patient-centered care. Jossey-Bass.

7. Gillespie, H., Kelly, M., Gormley, G., King, N., Gilliland, D., \& Dornan, T. (2018). How can tomorrow's doctors be more caring? A phenomenological investigation. Medical Education, 52(10), 1052-1063. https://doi.org/10.1111/medu.13684 
8. Grover, C., Mackasey, E., Cook, E., Nurse, H., Tremblay, L., Clinician, N., \& Loiselle, C. G. (2018). Patientreported care domains that enhance the experience of "being known" in an ambulatory cancer care centre. Canadian Oncology Nursing Journal, 28(3), 166-171.

https://doi.org/10.5737/23688076283166171

9. Hack, T. F., Degner, L. F., Watson, P., \& Sinha, L. (2006). Do patients benefit from participating in medical decision making? Longitudinal follow-up of women with breast cancer. Psycho-Oncology, 15(1), 9-19. https://doi.org/10.1002/pon.907

10. Li, C.-C., Matthews, A. K., Dossaji, M., \& Fullam, F. (2017). The Relationship of Patient-Provider Communication on Quality of Life among African-American and White Cancer Survivors. Journal of Health Communication, 22(7), 584-592. https://doi.org/10.1080/10810730.2017.1324540

11. Lee, T., Ko, I., Lee, I., Kim, E., Shin, M., Roh, S., Yoon, D., Choi, S., \& Chang, H. (2011). Effects of nurse navigators on health outcomes of cancer patients. Cancer Nursing, 34(5), 376-384. https://doi.org/10.1097/NCC.0b013e3182025007

12. Levesque, J.-F., Harris, M. F., \& Russell, G. (2013). Patient-centred access to health care: conceptualizing access at the interface of health systems and populations. International Journal for Equity in Health, 12(1), 18-26. https://doi.org/10.1186/1475-9276-12-18

13. Loiselle, C. G. (2019). Cancer information-seeking preferences are associated with distinct patient experiences and satisfaction with cancer care. Patient Education and Counseling, 102(6), 11871193. doi: 10.1016/j.pec.2019.01.009

14. Loiselle, C. G., Howell, D., Nicoll, I., \& Fitch, M. (2019). Toward the development of a comprehensive cancer experience measurement framework. Supportive Care in Cancer, 27(7), 2579-2589. doi:10.1007/s00520-018-4529-

15. Loiselle, C. G., Attieh, S., Cook, E., Tardif, L., Allard, M., Rousseau, C., Thomas, D., Saha-Chaudhuri, P., \& Talbot, D. (2020). The nurse pivot-navigator associated with more positive cancer care experiences and higher patient satisfaction. Canadian Oncology Nursing Journal, 30(1), 48-53. https://doi.org/10.5737/236880763014853

16. Loiselle, C. G., \& Brown, T. L. (2020). Increasing access to psychosocial oncology services means becoming more person-centered and situation-responsive. Supportive Care in Cancer, 28(12), 56015603. https://doi.org/10.1007/s00520-020-05659-8

17. Lyson, H. C., Haggstrom, D., Bentz, M., Obeng-Gyasi, S., Dixit, N., \& Sarkar, U. (2020). Communicating Critical Information to Cancer Survivors: an Assessment of Survivorship Care Plans in Use in Diverse Healthcare Settings. Journal of Cancer Education: The Official Journal of the American Association for Cancer Education. https://doi.org/10.1007/s13187-020-01725-1

18. National Research Corporation. (2003). Development and Validation of the Picker Ambulatory Oncology Survey Instrument in Canada. Lincoln, NE: Author

19. Pelzang, R. (2010). Time to learn: understanding patient-centred care. British Journal of Nursing (Mark Allen Publishing), 19(14), 912-917. https://doi.org/10.12968/bjon.2010.19.14.49050 
20. Rossy Cancer Network. (2019). The experience of patients with cancer at diagnosis and during treatment: A report on the results of the Rossy Cancer Network survey from 2014-2018. Rossy Cancer Network. https://www.mcgill.ca/rcr-rcn/files/rcr-rcn/rcn_patient_experience_report_2018.09.pdf

21. Scharp, K. M., \& Sanders, M. L. (2019). What is a theme? Teaching thematic analysis in qualitative communication research methods. Communication Teacher, 33(2), 117-121. https://doi.org/10.1080/17404622.2018.1536794

22. Schoen, C., Osborn, R., Huynh, P. T., Doty, M., Zapert, K., Peugh, J., \& Davis, K. (2005). Taking the pulse of health care systems: experiences of patients with health problems in six countries. Health Affairs (Project Hope), Supp/ Web Exclusives, W5. https://doi.org/10.1377/hlthaff.w5.509

23. Thórarinsdóttir, K., \& Kristjánsson, K. (2014). Patients' perspectives on person-centred participation in healthcare: A framework analysis. Nursing Ethics, 21(2), 129-147. https://doi.org/10.1177/0969733013490593

24. Tremblay, D., Roberge, D., Touati, N., Maunsell, E., \& Berbiche, D. (2017). Effects of interdisciplinary teamwork on patient-reported experience of cancer care. BMC Health Services Research, 17(1), 218. https://doi.org/10.1186/s12913-017-2166-7

25. Tzelepis, F., Sanson, F. R. W., Hall, A. E., Carey, M. L., Paul, C. L., \& Clinton, M. T. (2015). The quality of patient-centred care: Haematological cancer survivors' perceptions. Psycho-Oncology, 24(7), 796803. https://doi.org/10.1002/pon.3728

26. Wong, E. L. Y., Coulter, A., Hewitson, P., Cheung, A. W. L., Yam, C. H. K., Lui, S. fai, Tam, W. W. S., \& Yeoh, E. (2015). Patient experience and satisfaction with inpatient service: Development of short form survey instrument measuring the core aspect of inpatient experience. PLOS ONE, 10(4), 1-12. https://doi.org/10.1371/journal.pone.0122299\ 